\title{
Piecewise linear approximation to Fisher's equation
}

\author{
Z. Jovanoski ${ }^{1} \quad$ G. Robinson ${ }^{2}$
}

(Received 11 January 2012; revised 30 July 2012)

\begin{abstract}
A simple method is presented which allows the replacement of a nonlinear differential equation with a piecewise linear differential equation. The method is based on the idea that a curve of the nonlinear terms of the dependent variable in a differential equation can be replaced by an approximate curve consisting of a set of line segments tangent to the original curve. We apply this method to the ubiquitous Fisher's equation and demonstrate that accurate solutions are obtained with a relatively small number of line segments.
\end{abstract}

\section{Contents}

\section{Introduction}

C466

http://journal.austms.org.au/ojs/index.php/ANZIAMJ/article/view/5129 gives this article, (c) Austral. Mathematical Soc. 2012. Published August 5, 2012. ISSN 1446-8735. (Print two pages per sheet of paper.) Copies of this article must not be made otherwise available on the internet; instead link directly to this URL for this article. 
2 Approximating function

C467

3 Application to Fisher's equation

$\mathrm{C} 470$

3.1 Two segments . . . . . . . . . . . . . . . . . C470

3.2 Three segments . . . . . . . . . . . . . . . . C471

4 Comparison with exact solution

C473

5 Conclusion

C475

References

C476

\section{Introduction}

Formation and propagation of waves are sometimes described by a reactiondiffusion type partial differential equation (PDE), such as Fisher's equation. First introduced as a model for the propagation of a mutant gene [1], Fisher's equation has subsequently been used in population growth models [2], autocatalytic chemical reactions [3], and the healing of wounds [4].

Finding solutions to a nonlinear PDE is a difficult and challenging task. This certainly is true of Fisher's equation, even if it is the simplest of the reaction-diffusion equations - its nonlinearity is a simple quadratic,

$$
\frac{\partial u}{\partial t}=\frac{\partial^{2} u}{\partial x^{2}}+u(1-u) .
$$

The problem of finding exact solutions is often made easier by looking for travelling wave solutions. Assuming the wave is travelling in the positive direction, the substitution $\xi=x-$ ct reduces the original PDE to an ordinary differential equation (ODE),

$$
\frac{d^{2} u}{d \xi^{2}}+c \frac{d u}{d \xi}+u-u^{2}=0
$$


where $\mathbf{c}$ is the wave speed. Employing different techniques Ablowitz and Zeppetella [5] and Wang [6] obtained explicit travelling reaction front solutions for the special case $c=5 / \sqrt{6}$, namely

$$
u(\xi)=\frac{1}{\left(1+r e^{\xi / \sqrt{6}}\right)^{2}},
$$

where $r$ is an arbitrary positive constant arising from the translational symmetry of Fisher's PDE (1). The value of $r$ sets $u(\xi)$ at $\xi=0$. For example, the choice $r=\sqrt{2}-1$ ensures $u(0)=1 / 2$.

This article presents a method for finding an approximate solution to Fisher's equation. The basic idea is to replace the quadratic term by a piecewise linear function, thus replacing the nonlinear ODE by a set of linear inhomogeneous ODEs. The following sections outline the construction of the piecewise linear approximating function and its application to Fisher's equation. For illustrative purposes we use the special case of $c=5 / \sqrt{6}$, for which exact solutions exist, thus allowing estimates to be made of the accuracy of the piecewise approximation. However, the strength of the piecewise approximation lies in the fact that it may be applied to cases where an exact solution does not exist.

\section{Approximating function}

A travelling wave solution $\mathfrak{u}(\xi)$ of the ODE (2) is sought satisfying $0 \leqslant \mathfrak{u}(\xi) \leqslant$ 1 . The piecewise linear approximating function to Fisher's equation involves the replacement of the quadratic curve, $f(u)=u^{2}$ over the domain $0 \leqslant u \leqslant 1$, with a bounded polygonal line, as shown in Figure 1. If this approximating function consists of very many line segments, all of them tangent to the quadratic curve, the solution that is obtained approaches the exact solution of the nonlinear problem. In practice, only a few line segments tangent to the quadratic curve are sufficient for good accuracy (see Figure 1).

In the simplest case the piecewise linear function consists of two line segments, both tangent to $f(u)$, one at the end point $u=0$ and the other at the end 

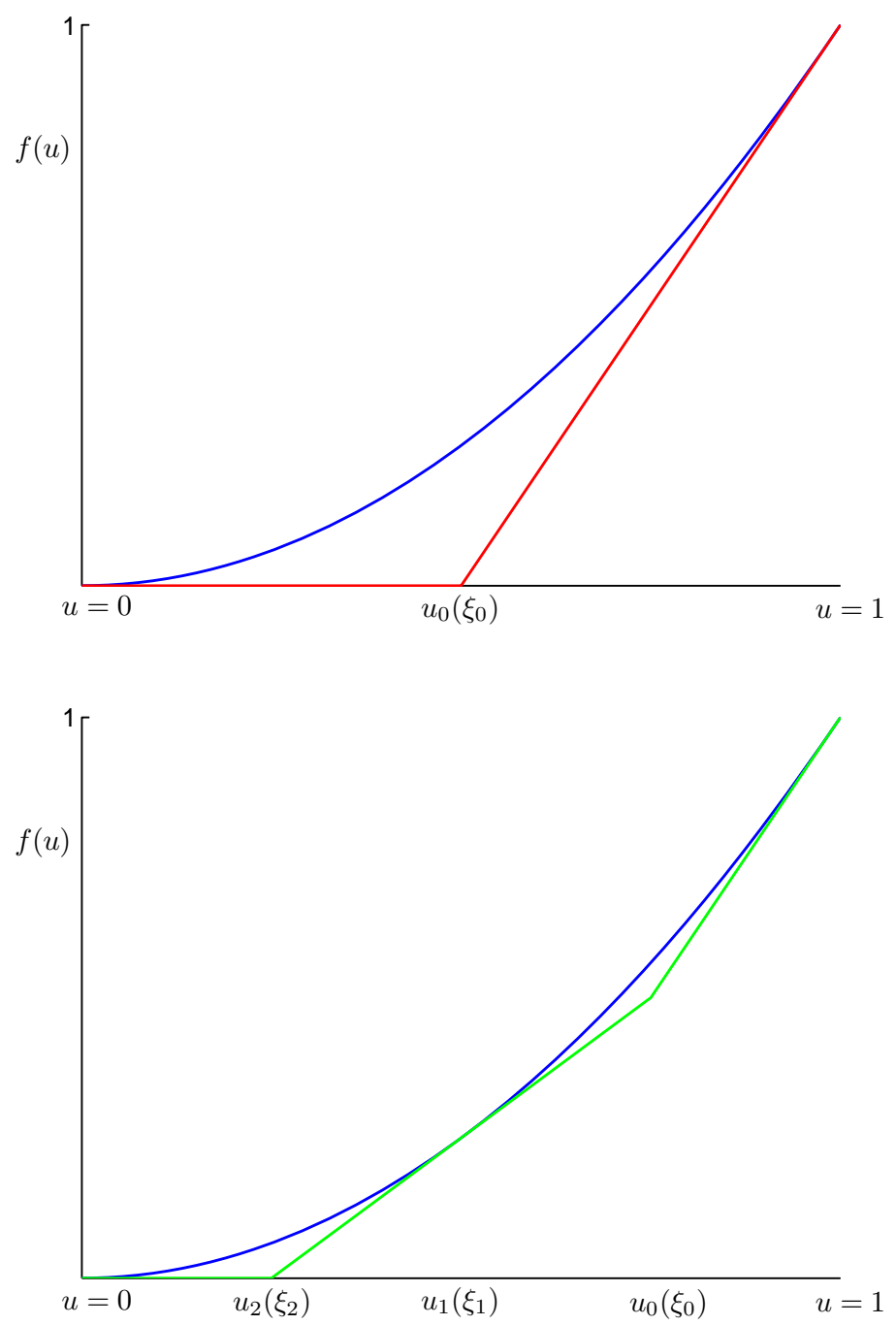

Figure 1: Plot of nonlinear term $f(u)=u^{2}$ with an approximating piecewise linear function consisting of two segments (top) and three segments (bottom). The approximating function $f_{\mathfrak{l}}(\mathfrak{u})$ is tangent to the curve at $\mathfrak{u}=0$ and $\mathfrak{u}=1$ for the two line segments and tangent to the curve at $\boldsymbol{u}=0, \mathfrak{u}_{1}\left(\xi_{1}\right)=\frac{1}{2}$ and $u=1$ for the three line segments. 
point $u=1$. The choices of $u=0$ and $u=1$, the fixed points of Fisher's equation, are used to ensure that the approximate solution has the correct asymptotic behaviour. In this case the approximate linear function $f_{l}(u)$ is

$$
f_{l}(u)= \begin{cases}0, & 0 \leqslant u \leqslant u_{0}, \\ 2 u-1, & u_{0} \leqslant u \leqslant 1 .\end{cases}
$$

Here $\mathfrak{u}_{0}\left(\xi_{0}\right)=1 / 2$ is the point of the intersection of the two line segments as depicted in the top plot of Figure 1.

In the case of three line segments, a middle line segment tangent to $f(u)$ at some interior point $\mathfrak{u}=\mathfrak{u}_{1}$ is added to the approximation (4). The resulting approximating function is

$$
f_{l}(u)= \begin{cases}0, & 0 \leqslant u \leqslant u_{2}, \\ 2 u_{1} u-u_{1}^{2}, & u_{2} \leqslant u \leqslant u_{0}, \\ 2 u-1, & u_{0} \leqslant u \leqslant 1\end{cases}
$$

The points $\mathfrak{u}_{2}\left(\xi_{2}\right)$ and $\boldsymbol{u}_{0}\left(\xi_{0}\right)$ are the points of intersection of the line segments as shown in the bottom plot of Figure 1. Each of these points is written in terms of $\mathfrak{u}_{1}\left(\xi_{1}\right)$, as $\mathfrak{u}_{2}=\mathfrak{u}_{1} / 2$ and $\mathfrak{u}_{0}=\left(\mathfrak{u}_{1}+1\right) / 2$.

For the three segments case, $\mathfrak{u}_{1}$ could be chosen at random or be specified. However, it is possible to choose $\mathfrak{u}_{1}$ to optimise the solution. There are several ways this might be done, one way is to choose $u_{1}$ to minimise $\int_{0}^{1}\left|u^{2}-f_{l}(u)\right| d u$. Breaking up the integral then integrating over each line segment and using a generalised version of Leibniz rule results in

$$
\frac{d}{d u_{1}} \int_{0}^{1}\left|u^{2}-f_{l}(u)\right| d u=\frac{u_{1}}{2}-\frac{1}{4} .
$$

Setting the right hand side to zero yields $\mathfrak{u}_{1}\left(\xi_{1}\right)=1 / 2$. It follows that $u_{2}\left(\xi_{2}\right)=1 / 4$ and $u_{0}\left(\xi_{0}\right)=3 / 4$. These optimal values are used in the analysis of the next section. 


\section{Application to Fisher's equation}

The PDE (1) possesses a stable state at $\boldsymbol{u}=1$ and an unstable state at $\boldsymbol{u}=0$. The front propagating at a constant speed $c$ into the the unstable state is expressed by the ODE (2). With $c=5 / \sqrt{6}$, the explicit solution (3) represents a wave moving at a constant speed in the positive direction satisfying the boundary conditions $u \rightarrow 1$ as $\xi \rightarrow-\infty$ and $u \rightarrow 0$ as $\xi \rightarrow \infty$. Further, the solution passing through $u(0)=1 / 2$ requires that $r=\sqrt{2}-1$.

Our aim is to apply the piecewise approximating function with two and three segments to the ODE (2).

\subsection{Two segments}

Substituting the approximation (4) for the nonlinear term in the ODE (2) leads to

$$
\frac{d^{2} u}{d \xi^{2}}+c \frac{d u}{d \xi}= \begin{cases}-u, & 0 \leqslant u \leqslant \frac{1}{2} \\ u-1, & \frac{1}{2} \leqslant u \leqslant 1\end{cases}
$$

The solution to this piecewise linear second-order ODE is

$$
u(\xi)= \begin{cases}\mathrm{A} e^{\sigma_{+} \xi}+\mathrm{B} e^{\sigma_{-} \xi}, & \xi \geqslant \xi_{0}, \\ 1+\mathrm{Ce}^{\lambda_{+} \xi}+\mathrm{D} e^{\lambda_{-} \xi}, & \xi \leqslant \xi_{0},\end{cases}
$$

where

$$
\sigma_{ \pm}=\frac{-c \pm \sqrt{c^{2}-4}}{2}, \quad \lambda_{ \pm}=\frac{-c \pm \sqrt{c^{2}+4}}{2} .
$$

The constants $\mathrm{A}, \mathrm{B}, \mathrm{C}, \mathrm{D}$ and $\xi_{0}$ all need to be determined. Requiring $u \rightarrow 1$ as $\xi \rightarrow-\infty$, implies $D=0$. By construction $u\left(\xi_{0}\right)=1 / 2$ (see Figure 1 ). Continuity of $u(\xi)$ at $\xi_{0}$ leads to

$$
\mathrm{A} e^{\sigma_{+} \xi_{0}}+\mathrm{Be}^{\sigma_{-} \xi_{0}}=\frac{1}{2}, \quad \mathrm{C} e^{\lambda_{+} \xi_{0}}=-\frac{1}{2} .
$$


Similarly, continuity of the derivative of $\mathfrak{u}(\xi)$ at $\xi_{0}$ leads to

$$
\mathrm{A} \sigma_{+} e^{\sigma_{+} \xi_{0}}+B \sigma_{-} e^{\sigma_{-} \xi_{0}}=C \lambda_{+} e^{\lambda_{+} \xi_{0}} .
$$

These equations establish the constants

$$
A=\frac{1}{2}\left(\frac{1}{2}-\delta\right), \quad B=-\frac{1}{2}\left(\frac{1}{2}+\delta\right), \quad \delta=\frac{-2 c+\sqrt{c^{2}+4}}{2 \sqrt{c^{2}-4}} .
$$

For $\delta$ to be real and finite requires $c>2$ for a wave travelling in the positive direction. The only remaining free parameter is $\xi_{0}$. Setting $\xi_{0}=0$ enables direct comparison with the explicit solution (3). For the case $c=5 / \sqrt{6}$,

$$
u(\xi)= \begin{cases}e^{-2 \xi / \sqrt{6}}-\frac{1}{2} e^{-3 \xi / \sqrt{6}}, & \xi \geqslant 0, \\ 1-\frac{1}{2} e^{\xi / \sqrt{6}}, & \xi \leqslant 0 .\end{cases}
$$

This form of the solution was first obtained by Rinzel and Keller [7] for propagation of a nerve impulse.

\subsection{Three segments}

Using the approximation (5) the travelling front ODE becomes

$$
\frac{d^{2} u}{d \xi^{2}}+c \frac{d u}{d \xi}= \begin{cases}-u, & 0 \leqslant u \leqslant \frac{1}{4} \\ -\frac{1}{4}, & \frac{1}{4} \leqslant u \leqslant \frac{3}{4} \\ u-1, & \frac{3}{4} \leqslant u \leqslant 1\end{cases}
$$

The solution over each line segment is

$$
u(\xi)= \begin{cases}A e^{\sigma_{+} \xi}+B e^{\sigma_{-} \xi}, & \xi \geqslant \xi_{2}, \\ -\frac{1}{4 c} \xi+C+D e^{-c \xi}, & \xi_{0} \leqslant \xi \leqslant \xi_{2}, \\ 1+E e^{\lambda_{+} \xi}+F e^{\lambda_{-} \xi}, & \xi \leqslant \xi_{0} .\end{cases}
$$


Requiring $u \rightarrow 1$ as $\xi \rightarrow-\infty$, implies $F=0$. Continuity of $u(\xi)$ at $\xi_{0}$ requires

$$
\begin{aligned}
\mathrm{Ee}^{\lambda_{+} \xi_{0}} & =-\frac{1}{4}, \\
-\frac{1}{4 \mathrm{c}} \xi_{0}+\mathrm{C}+\mathrm{D} e^{-\mathrm{c} \xi_{0}} & =\frac{3}{4} .
\end{aligned}
$$

Continuity of the derivative of $u(\xi)$ at $\xi_{0}$ requires

$$
\mathrm{E} \lambda_{+} \mathrm{e}^{\lambda_{+} \xi_{0}}=-\frac{1}{4 \mathrm{c}}-\mathrm{cD} e^{-\mathrm{c} \xi_{0}} .
$$

Substituting for E entails

$$
\mathrm{D}=\left(\lambda_{+} \mathrm{c}-1\right) \frac{e^{\mathrm{c} \xi_{0}}}{4 \mathrm{c}^{2}}
$$

Since $u\left(\xi_{1}\right)=1 / 2$, setting $\xi_{1}=0$ gives $C+D=1 / 2$. Continuity of $u(\xi)$ and its derivative at $\xi_{2}$ yields

$$
\begin{aligned}
\mathrm{A} e^{\sigma_{+} \xi_{2}}+\mathrm{Be}^{\sigma_{-} \xi_{2}} & =\frac{1}{4}, \\
-\frac{1}{4 \mathrm{c}} \xi_{2}+\mathrm{C}+\mathrm{D} e^{-\mathrm{c} \xi_{2}} & =\frac{1}{4},
\end{aligned}
$$

and

$$
\mathrm{A} \sigma_{+} e^{\sigma_{+} \xi_{2}}+\mathrm{B} \sigma_{-} e^{\sigma_{-} \xi_{2}}=\frac{1}{4}=-\frac{1}{4 \mathrm{c}}-\mathrm{cD} e^{-\mathrm{c} \xi_{2}} .
$$

Multiplying Equation (11) by $\sigma_{+}$(and likewise by $\sigma_{-}$) then subtraction of Equation (13) leads to

$$
\begin{aligned}
& \frac{\sigma_{-}}{4}+\frac{1}{4 c}+\mathrm{cD} e^{-c \xi_{2}}=\mathrm{A}\left(\sigma_{-}-\sigma_{+}\right) e^{\sigma_{+} \xi_{2}} \\
& \frac{\sigma_{+}}{4}+\frac{1}{4 c}+\mathrm{cD} e^{-c \xi_{2}}=\mathrm{B}\left(\sigma_{+}-\sigma_{-}\right) e^{\sigma_{-} \xi_{2}} .
\end{aligned}
$$


Substituting for D and then solving for $A$ and $B$ results in

$$
\begin{aligned}
& A=\frac{e^{-\sigma_{+} \xi_{2}}}{4 c\left(\sigma_{-}-\sigma_{+}\right)}\left[\sigma_{-} c+\left(\lambda_{+} c-1\right) e^{c\left(\xi_{0}-\xi_{2}\right)}+1\right], \\
& \mathrm{B}=\frac{\mathrm{e}^{-\sigma_{-} \xi_{2}}}{4 \mathrm{c}\left(\sigma_{+}-\sigma_{-}\right)}\left[\sigma_{+} \mathrm{c}+\left(\lambda_{+} \mathrm{c}-1\right) \mathrm{e}^{\mathrm{c}\left(\xi_{0}-\xi_{2}\right)}+1\right] .
\end{aligned}
$$

Further, substituting for $\mathrm{C}$ in Equations (10) and (12), then adding and subtracting them leads to two coupled algebraic equations relating $\xi_{0}$ and $\xi_{2}$, namely

$$
\begin{aligned}
-c\left(\xi_{0}-\xi_{2}\right)+\left(\lambda_{+} c-1\right)\left(1-e^{c\left(\xi_{0}-\xi_{2}\right)}\right)-2 c^{2} & =0, \\
-c\left(\xi_{0}+\xi_{2}\right)+\left(\lambda_{+} c-1\right)\left(1+e^{c\left(\xi_{0}-\xi_{2}\right)}-2 e^{c \xi_{0}}\right) & =0 .
\end{aligned}
$$

Letting $\bar{\xi}=\xi_{0}-\xi_{2}$ and for the special case $c=5 / \sqrt{6}$ these equations reduce to

$$
\begin{aligned}
5 \sqrt{6} \bar{\xi}-e^{5 \bar{\xi} / \sqrt{6}}+51 & =0, \\
5 \sqrt{6}\left(2 \xi_{0}-\bar{\xi}\right)+e^{5 \bar{\xi} / \sqrt{6}}-2 e^{5 \xi_{0} / \sqrt{6}}+1 & =0 .
\end{aligned}
$$

From the first of these equation $\bar{\xi} \approx-4.16$, then from the second $\xi_{0} \approx-2.12$. The complete solution over the three segments is then

$$
u(\xi)= \begin{cases}\frac{1}{20}\left[\left(9+e^{5 \bar{\xi} / \sqrt{6}}\right) e^{-2\left(\xi-\xi_{2}\right) / \sqrt{6}}\right. & \\ \left.-\left(4+e^{5 \bar{\xi} / \sqrt{6}}\right) e^{-3\left(\xi-\xi_{2}\right) / \sqrt{6}}\right], & \xi \geqslant \xi_{2}, \\ \frac{1}{2}-\frac{\sqrt{6}}{20} \xi-\frac{1}{100}\left(e^{-5\left(\xi-\xi_{0}\right) / \sqrt{6}}-e^{5 \xi_{0} / \sqrt{6}}\right), & \xi_{0} \leqslant \xi \leqslant \xi_{2}, \\ 1-\frac{1}{4} e^{\left(\xi-\xi_{0}\right) / \sqrt{6}}, & \xi \leqslant \xi_{0} .\end{cases}
$$

\section{Comparison with exact solution}

Figure 2 shows the result of the piecewise linear approximation applied to Fisher's equation compared with the exact solution (3). The upper plot shows 

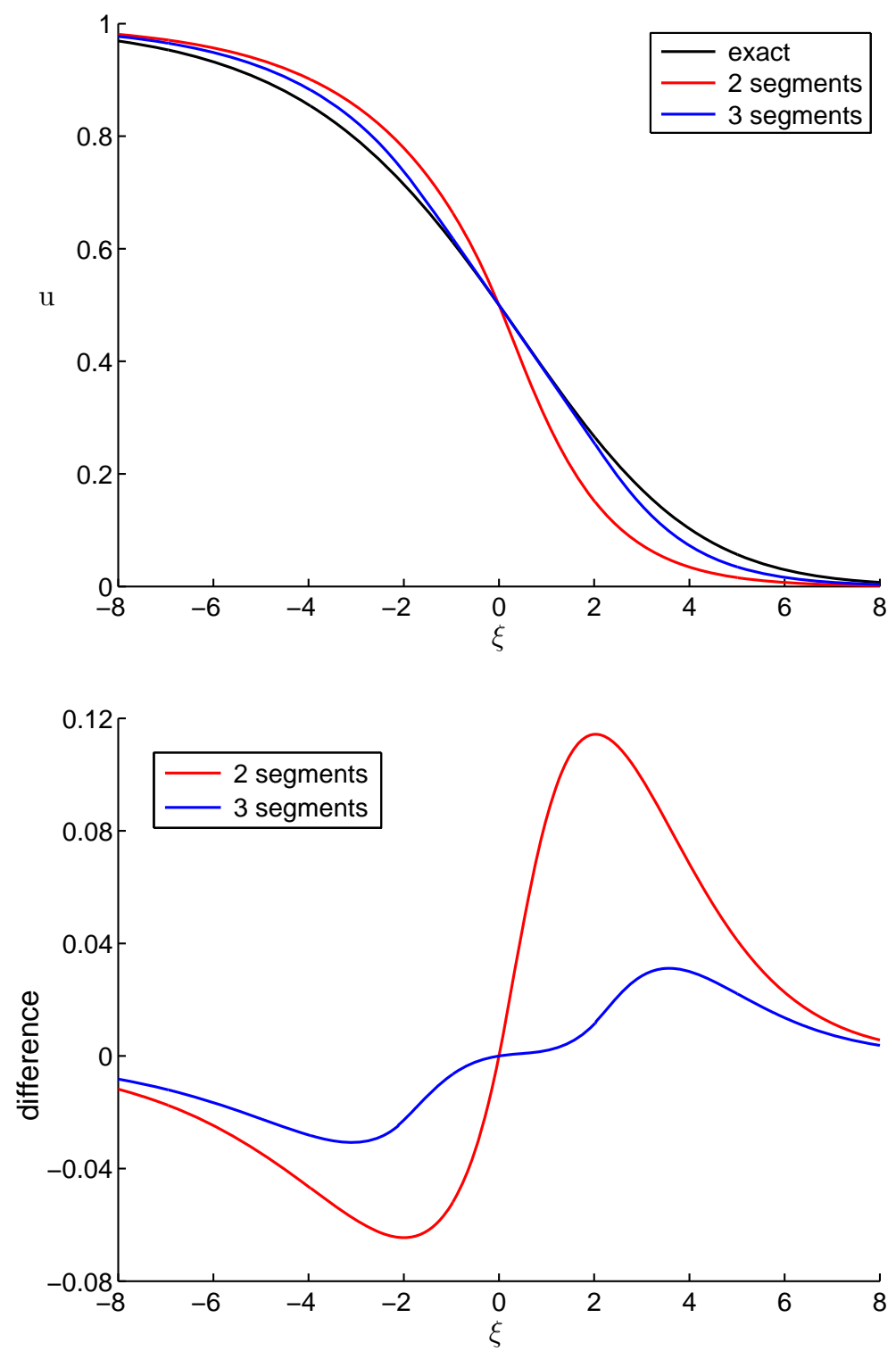

Figure 2: The exact (3) and approximate solutions (7) and (18) to Fisher's equation for the special case of $c=5 / \sqrt{6}$ are presented in the top plot. The difference between them is depicted in the bottom plot. 
the approximate solutions are quite good. Employing two segments provides a reasonable solution; however, using three segments offers a much improved solution. Both solutions have the correct asymptotic behaviour. The bottom plot illustrates the difference between the exact solution and the two and three segment solutions. As expected, the three segments solution is a better approximation over the entire domain. Using two segments the maximum error is about $12 \%$. The error falls sharply to about $3 \%$ using three segments.

\section{Conclusion}

The idea of approximating the nonlinear term in a PDE or ODE with a piecewise linear function is conceptually simple. What seems rather remarkable is the high level of accuracy achieved even in the case of as few as three line segments [8]. The method is used to find an approximate solution for the important Fisher's equation. Solutions based on two and three line segments are derived and compared to the exact travelling wave solution with speed $c=5 / \sqrt{6}$. Using three line segments for the approximating function the error is less than $3 \%$ over the entire domain.

The only drawback to the method described is that the value of $c$ must be specified a priori. Since for Fisher's equation (1) there exists a continuous family of travelling wave solutions, the question of selection of the wave front speed arises, as the travelling wave solutions assume all wave speeds $c \geqslant 2$. A positive initial profile $\mathfrak{u}(x, 0)$, decaying at least exponentially as $x \rightarrow \infty$, evolves into a travelling wave. Extensive numerical simulations of Fisher's equation (1) demonstrate that if $u(x, 0) \sim e^{-\beta x}$ as $x \rightarrow \infty$, then the initial profile evolves to a wave front of speed [9]

$$
c(\beta)= \begin{cases}\left(1+\beta^{2}\right) / \beta, & \beta \leqslant 1, \\ 2, & \beta \geqslant 1 .\end{cases}
$$

We note from Equations (6) and (8) that the solution should evolve to a wave speed $c(\beta)$ given by (19) for $\beta=-\sigma_{+}$. 


\section{References}

[1] R. A. Fisher. The wave of advance of advantageous genes. Ann. of Eugenics., 7, 355-369, 1937. C466

[2] V. M. Kenkre and M. N. Kuperman. Applicability of the Fisher equation to bacterial population dynamics. Phys. Rev. E., 67, 2003, 051921. doi:10.1103/PhysRevE.67.051921 C466

[3] D. A. Kessler, Z. Ner and L. M. Sander. Front propagation: precursors, cutoffs, and structural stability. Phys. Rev. E., 58, 1998, 107-114. C466

[4] P. K. Maini, D. L. S. McElwain and D. I. Leavesley. Traveling wave model to interpret a wound-healing cell migration assay for human peritoneal mesothelial cells. Tissue Eng., 10, 2004, 475-482. C466

[5] M. J. Ablowitz and A. Zeppetella. Explicit solutions of Fisher's equation for a special wave speed. Bull. Math. Biol., 41, 1979, 835-840. C467

[6] X. Y. Wang. Exact and explicit solitary wave solutions for the generalised Fisher equation. Phys. Lett. A, 131, 1988, 277-279. C467

[7] J. Rinzel and J. B. Keller. Traveling wave solutions of a nerve conduction equation. Biophys. J., 13, 1973, 1313-1337. C471

[8] Z. Jovanoski and S. Soo. Piecewise linear approximation of nonlinear ordinary differential equations. ANZIAM J. (E), 51, 2010, C570-C585. http://journal. austms.org.au/ojs/index.php/ANZIAMJ/article/ view/2622 C475

[9] T. Hagstrom and H. B. Keller. The numerical calculation of traveling wave solutions of nonlinear parabolic equations. SIAM J. Sci. Stat. Comput., 7, 1986, 978-988. C475 


\section{Author addresses}

1. Z. Jovanoski, Applied and Industrial Mathematics Research Group, School of Physical, Environmental and Mathematical Sciences, University of New South Wales, Canberra 2600, Australia. mailto:z.jovanoski@adfa.edu.au

2. G. Robinson, School of Physical, Environmental and Mathematical Sciences, University of New South Wales, Canberra 2600, Australia. mailto:g.robinson@adfa.edu.au 